\title{
Lesão por Pressão relacionada a Dispositivos Médicos em Unidade de Terapia Intensiva
}

\author{
Pressure Injury related to Medical Devices in the Intensive Care Unit
}

Lesión por Presión relacionada con Dispositivos Médicos en la Unidad de Cuidados Intensivos

Shirley Junqueira Barbosa ${ }^{1 *}$, Sheila Carminati de Lima Soares ${ }^{1}$, Amanda Gabrielle Silva Queiroz ${ }^{1}$, Rafael Zondonadi de Souza', Sara Ferreira Sottocornola'.

\section{RESUMO}

Objetivo: Avaliar a ocorrência de Lesão por Pressão Relacionada a Dispositivos Médicos (LPRDM) em uma UTI adulto. Métodos: Trata-se de um estudo de campo observacional, prospectivo de caráter quantitativo realizado com 33 pacientes submetidos à utilização de dispositivos médicos durante a internação. $O$ estudo foi aprovado pelo Comitê de Ética em Pesquisa respeitando critérios éticos e legais. Resultados: Houve uma incidência de LPRDM em $84,8 \%$ dos pacientes analisados, tendo como os principais dispositivos envolvidos o TOT (51\%), SVD (16\%) e SNE (12\%), sendo os principais locais acometidos região de orelha, boca e nariz. Dentre as principais medidas de prevenção foi observado aplicação de gazes em pontos de pressão causados por dispositivos respiratórios e medidas de tratamento a descompressão, reposicionamento do dispositivo acima da lesão e aplicação de coberturas para evolução da ferida. Conclusão: Verificou-se por meio dessa pesquisa um alto índice de ocorrência de LPRDM, o que indica a necessidade de intervenções da equipe de enfermagem para a redução das mesmas e melhora na qualidade da assistência, visto que a equipe de enfermagem possui um papel imprescindível no processo do cuidar.

Palavras-chave: Equipamentos e provisões, Segurança do paciente, Lesão por pressão, Cuidados de enfermagem, Cuidados críticos.

\begin{abstract}
Objective: Evaluate the occurrence of Medical Device Related Pressure Injuries (MDRPI) in an adult ICU. Methods: This is an observational, prospective field study of a quantitative nature carried out with 33 patients undergoing the use of medical devices during hospitalization. The study was approved by the Research Ethics Committee respecting ethical and legal criteria. Results: There was an incidence of LPRDM in $84.8 \%$ of the patients analyzed, the main devices involved being the TOT (51\%), SVD (16\%) and SNE (12\%), with the main sites affected in the region of ear, mouth and nose. Among the main prevention measures was the application of gauze at pressure points caused by respiratory devices and treatment measures such as decompression, repositioning of the device above the lesion and application of dressings for wound evolution. Conclusion: It was found through this research a high rate of occurrence of LPRDM, which indicates the need for interventions by the nursing team to reduce them and improve the quality of care, since the nursing team has an essential role in the process of caring.
\end{abstract}

Key words: Equipment and supplies, Patient safety, Pressure injury, Nursing care, Critical care.

\footnotetext{
${ }^{1}$ Hospital Regional da Cacoal (HRC), Cacoal - RO.

*E-mail: shirley.jjunqueira@gmail.com
}

SUBMETIDO EM: 11/2021 


\begin{abstract}
RESUMEN
Objetivo: Evaluar la aparición de Lesiones por Presión Relacionadas con Dispositivos Médicos (LPRDM) en una UCl para adultos. Métodos: Se trata de un estudio de campo, observacional, prospectivo y de carácter, realizado con 33 pacientes que toman uso cuantitativo de dispositivos médicos durante la hospitalización. El estudio fue aprobado por el Comité de Ética en Investigación y cumplió con criterios de investigación éticos y legales. Resultados: Hubo una incidencia de LPRDM en el $84,8 \%$ de los pacientes analizados, siendo los principales dispositivos implicados el TOT (51\%), SVD (16\%) y SNE (12\%), siendo los principales sitios af ectados el oído, la boca y nariz. Entre las principales medidas de prevención estuvo la aplicación de gasas en puntos de presión provocados por dispositivos respiratorios y medidas de tratamiento como descompresión, reposicionamiento del dispositivo sobre la lesión y aplicación de apósitos para la evolución de la herida. Conclusión: Se encontró a través de esta investigación una alta tasa de ocurrencia de LPRDM, lo que indica la necesidad de intervenciones por parte del equipo de enfermería para reducirlas y mejorar la calidad de la atención, ya que el equipo de enfermería tiene un rol fundamental en el proceso de cuidar.
\end{abstract}

Palabras clave: Equipos y suministros, Seguridad del paciente, Lesiones por presión, Cuidados de enfermería, Cuidados intensivos.

\title{
INTRODUÇÃO
}

As Lesões Por Pressão (LPP) são definidas como danos causados na pele ou em tecidos moles subjacentes gerados poruma pressão intensa e/ou prolongada sobre uma proeminência óssea ou relacionada a dispositivos médicos, apresentando-se em forma de pele intacta ou úlcera aberta. São consideradas como os principais eventos adversos e incidentes que ocorrem em uma Unidade de Terapia Intensiva (UTI) levando em consideração que pacientes críticos são considerados ainda mais vulneráveis para o desenvolvimento dessas lesões devido fatores intrínsecos e extrínsecos como imobilidade no leito, idade avançada, uso de sedação, comorbidades associadas, uso de drogas vasoativas, estado nutricional debilitado e procedimentos invasivos (EDSBERG LE, et al., 2016; BORGHARDT AT, et al., 2016; COYER F e TAYYIB N, 2017; ALI YCMM, et al., 2020).

Diante do pressuposto, observa-se que diversas pesquisas foram desenvolvidas ao longo dos anos com ênfase às lesões por decúbito conhecidas como "lesões clássicas": sacrais; tro cânteas; calcâneas; occipitais e outras; e diante da ênfase direcionada a esta modalidade de lesão, culminou-se em uma redução na incidência e prevalência nas unidades de saúde. No entanto, no que se refere à Lesões por Pressão Relacionadas a Dispositivos Médicos (LPRDM), consideradas atualmente epidemia em saúde devido ao aumento das ocorrências dentro das UTIs, chega-se à conclusão que ainda é um tema limitado na literatura no que se refere a relevância, frequência, locais de desenvolvimento, forma de estadiamento, prevenção, tratamento, locais de ocorrência e os dispositivos envolvidos (ASTI E, et al., 2017; DELMORE BA e AYELLO EA, 2017; CAVALCANTI EO e KAMADA I, 2020).

As LPRDM foram incluídas no ano de 2016 pelo National Pressure Ulcer Advisory Painel (NPUAP) em suas novas diretrizes definindo-as oficialmente como lesões resultantes do uso de dispositivos projetados para fins diagnósticos e terapêuticos, apresentando-se no formato do dispositivo envolvido (EDSBERG LE, et al., 2016). Essas lesões são ocasionadas pelo uso prolongado de dispositivos médicos em contato direto com a pele ou mucosas com pressões maiores que $32 \mathrm{mmHg}$ capazes de resultar em uma oclusão do fluxo sanguíneo gerando alterações isquêmicas (ASTI E, et al., 2017; BEZERRA SMG, et al., 2020).

Estudo evidenciou que pacientes em uso contínuo de Dispositivos Médicos (DM) em UTI num período de 24 horas apresentaram ocorrência de $11,8 \%$ de LPRDM, no quarto dia houve elevação para $48 \%$ enquanto no décimo dia observou-se $82,3 \%$, concluindo que quanto maior o tempo de internação em uso de dispositivos, maior o índice de LPRDM (HANONU S e KARADAG A, 2016).

Os principais fatores que propiciam esta modalidade de lesões são o contato direto do dispositivo com a pele ou mucosa, características dos materiais utilizados, forma de fixação, dificuldade no ajuste ao corpo do 
paciente, não verificação diária dos dispositivos, falta de conscientização da equipe sobre os riscos, tempo de uso prolongado, pacientes sedados ou confusos, umidade e integridade da pele e por fim, o material geralmente rígido desses dispositivos (BARAKAT-JOHNSON M, et al., 2017; KAYSER SA, et al., 2018; CAVALCANTI EO e KAMADA I, 2020).

Os principais dispositivos de risco citados pela literatura no desenvolvimento dessas lesões são o colar cervical, dispositivos respiratórios, sondas nasogástricas e nasoentéricas, meias antiembolismo, aparelhos ortopédicos, cateteres uretrais, restrições de pulso, gessos, talas, dispositivos protéticos, cateteres peridurais, cateteres uretrais e oxímetro depulso (ARNOLD-LONG M, et al., 2017; BARAKAT-JOHNSON M, et al., 2017).

Em um estudo mineiro realizado no ano de 2018, constatou que o custo gerado semestralmente no tratamento de lesões por pressão por paciente internado foi de $R \$ 1.886,00$ e o custo total foi de $R \$$ 113.186,006, destacando ainda mais que as estratégias de prevenção geram um custo benefício tanto ao paciente quanto às instituições de saúde, uma vez que são capazes de reduzir o tempo de internação hospitalar, reduz a carga de trabalho da equipe de enfermagem, melhora a qualidade de vida e assistência prestada ao paciente e reflete nos indicadores de incidência e prevalência de LPPs nas unidades de saúde (DONOSO MTV, et al., 2019).

É importante destacar o impacto que as LPRDM geram ao paciente crítico e à assistência, causam desconforto, dor, risco de infecção, prolongam o tempo de permanência do paciente na UTI, promovem a sobrecarga de trabalho da equipe de enfermagem e consequentemente geram altos custos para as instituições de saúde quando comparadas às lesões de decúbito. Na maioria das vezes não é um tema apreciado pela equipe de saúde e na literatura, evidenciando ainda mais a neces sidade de desenvolvimento de estudos acerca deste tema tão importante (ASTI E, et al., 2017; ALI YCMM, et al., 2020).

Diante do pressuposto, esta pesquisa teve como objetivo avaliar a ocorrência de lesão por pressão relacionada a dispositivos médicos em uma UTI adulto do interior do Estado de Rondônia.

\section{MÉTODOS}

Pesquisa de campo utilizando-se do método observacional, prospectivo de caráter quantitativo realizado em hospital referência para a II Macrorregião de Saúde do estado de Rondônia que conta com 2 UTls adulto (18 leitos) e taxa de rotatividade semestral em torno de 150 pacientes em ambas. Para local da pesquisa foi selecionada a UTI 2 (atualmente Unidade COVID 4) que conta com 8 leitos.

Para guiar este estudo, foram investigadas as seguintes variáveis: taxa de ocorrência de LPRDM, dispositivos médicos envolvidos em cada caso, locais de desenvolvimento das LPRDM e medidas de prevenção e tratamento utilizadas.

No período proposto pela pesquisa, foram admitidos 40 pacientes no setor sendo a amostra composta por 33 pacientes internados na UTI 2 (Unidade COVID 4) no período de 21 de fevereiro a 21 de abril do ano de 2021. A coleta foi realizada por meio de visitas no período vespertino com frequência de três visitas por semana não necessariamente consecutivas com duração média de uma hora, sendo realizado neste p eríodo a inspeção da pele abaixo dos dispositivos para verificar a ocorrência de sinais compatíveis com LPRDM.

Utilizaram-se como critérios de exclusão pacientes já admitidos com LPRDM e como critério de inclusão todos os pacientes admitidos durante o período delimitado sem lesões dessa modalidade submetidos à utilização de um ou mais dos seguintes dispositivos médicos: Tubo Orotraqueal (TOT); Máscara de Venturi; Máscara com Reservatório; Ventilação Não Invasiva (VNI); Cateter Venoso Central (CVC); Traqueo stomia (TQT); Sonda Nasoenteral (SNE); Manguito de Pressão Arterial; Oxímetro de Pulso; Sonda Vesical de Demora (SVD).

O Projeto de pesquisa foi submetido para apreciação do Comitê de Ética em Pesquisa (CEP) no mês de novembro de 2020 obtendo autorização sob parecer CAAE nํ 40368220.1.0000.5298. Para realização desta pesquisa foram utilizados dados obtidos a partir da observação e inspeção da pele e mucosas de pacientes sob os dispositivos médicos em uso com risco mínimo de desconforto ao paciente. 
A busca para o referencial teórico contou com as seguintes bases de dados: Literatura Latino Americana e do Caribe em Ciências da Saúde (Lilacs), Medical Literature Analysis and Retrieval System Online (Medline), Base de Dados de Enfermagem (BDENF) e Scientific Electronic Library Online (SciELO) sendo acessadas por meio da Biblioteca Virtual de Saúde (BVS) e da Biblioteca Virtual Public/Publisher Medline (PubMed) a partir dos Descritores em Ciências da Saúde (DeCS) "Equipamentos e Provisões ", "Segurança do pacie nte", "Lesão por Pressão", "Cuidados de Enfermagem" e "Cuidados Críticos".

\section{RESULTADOS}

Considerando os critérios de inclusão e exclusão, foram selecionados 33 (100\%) pacientes. Houve uma incidência de LPRDM em 84,8\% (28) dos pacientes internados neste período e em apenas 15,2\% (5) casos não houve desenvolvimento. Diante dos dispositivos delimitados a serem investigados pelo estudo, foi evidenciado um total de 122 LPRDM sendo os dispositivos envolvidos: Tubo Orotraqueal (51\%); SVD (16\%); SNE (12\%); Manguito de Pressão Arterial (10\%); Oxímetro de pulso (9\%); Cateter Nasal do tipo óculos (2\%) (Tabela 1).

Tabela 1 - Distribuição dos dispositivos de risco para desenvolvimento das Lesões por Pressão Relacionadas a Dispositivos Médicos em UTI adulto.

\begin{tabular}{lcc}
\hline Dispositivos causadores das LPRDM & Total & $\%$ \\
\hline Manguito de pressão arterial & 12 & $10 \%$ \\
Sonda Nasoenteral & 15 & $12 \%$ \\
Cateter nasal & 2 & $2 \%$ \\
Sonda Vesical de Demora & 20 & $16 \%$ \\
Oxímetro de pulso & 11 & $9 \%$ \\
Tubo Orotraqueal & 62 & $51 \%$ \\
TOTAL & 122 & $100 \%$ \\
\hline
\end{tabular}

Fonte: Barbosa SJ, et al., 2021.

Diante do pressuposto, à partir da avaliação dos dispositivos causadores das LPRDM, houve uma incidência total de 122 lesões que foram distribuídas por locais anatômicos. Observou-se uma predominância de lesões em região de orelha $(37,7 \%)$ e boca (13,9\%) (Gráfico1).

Gráfico 1 - Distribuição dos locais anatômicos acometidos por Lesões por Pressão Relacionadas a Dispositivos Médicos em UTI adulto.

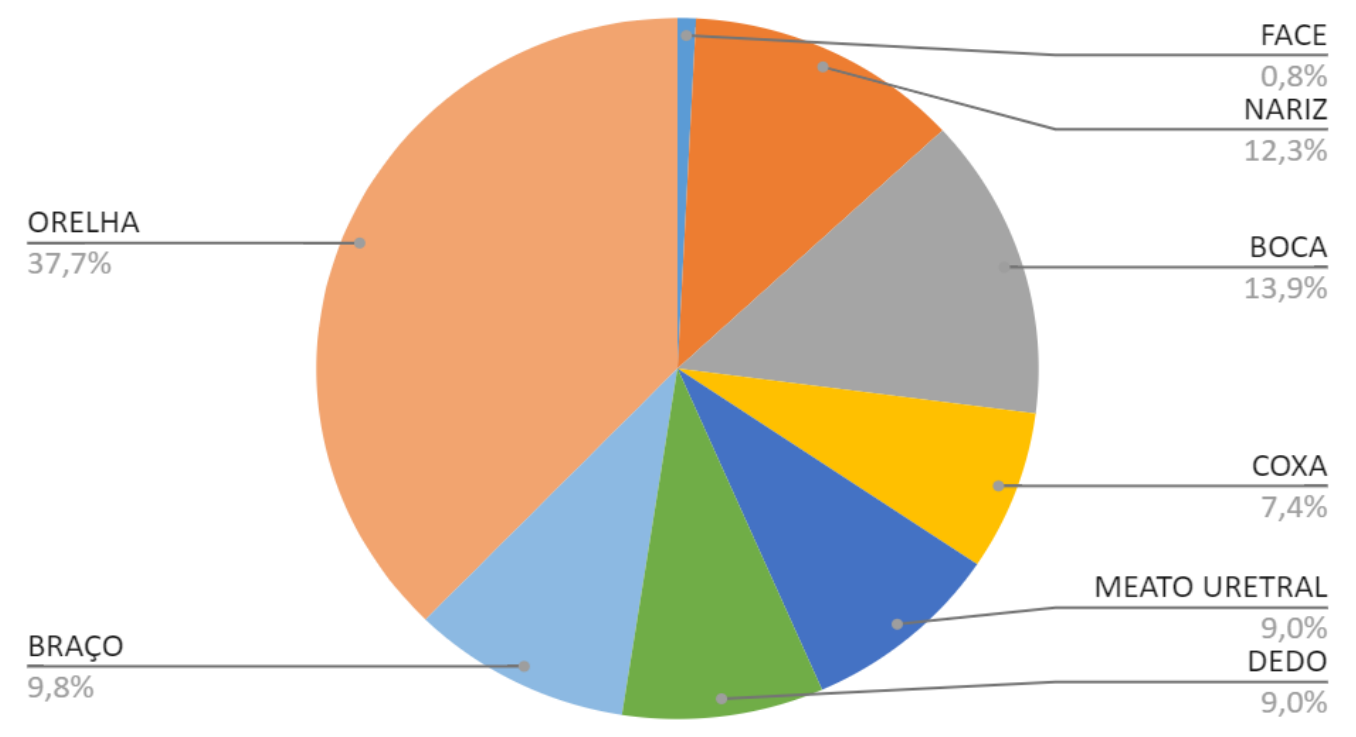

Fonte: Barbosa SJ, et al., 2021. 
A partir da ocorrência de lesões, avaliação dos principais locais acometidos e dispositivos relacionados, também foram observadas as principais medidas aplicadas pela equipe de enfermagem no processo de prevenção das LPRDM e tratamento utilizado em pacientes acometidos por essas lesões (Quadro1).

Quadro 1 - Medidas de prevenção e tratamento utilizadas nos pacientes acometidos por Lesões por Pressão Relacionadas a dispositivos médicos em UTI adulto.

\begin{tabular}{|c|c|}
\hline $\begin{array}{r}\text { didas de prevenção a } \\
\text { Enfern }\end{array}$ & $\begin{array}{r}\text { Medidas de tratamento } \\
\text { Enfer }\end{array}$ \\
\hline $\begin{array}{l}\text { - Aplicação de gaze em pontos de pressão na } \\
\text { fixação do TOT. } \\
\text { - Acolchoamento utilizando-se de gaze estéril } \\
\text { em fixação de cânula de TQT. }\end{array}$ & $\begin{array}{l}\text { - Descompressão e reposicionamento do } \\
\text { dispositivo acima da lesão. } \\
\text { - Aplicação de coberturas adequadas para boa } \\
\text { evolução da ferida aberta. }\end{array}$ \\
\hline
\end{tabular}

Fonte: Barbosa SJ, et al., 2021.

\section{DISCUSSÃO}

A análise dos dados agrupados traz um alerta para o alto índice de ocorrência de LPRDM de $84,8 \%$ sendo evidenciado em média 3,6 lesões por paciente internado. Foram salientadas as seguintes fragilidades durante o período observacional: tração excessiva na fixação do TOT; tração excessiva na fixação da SNE; ausência de fixação de SVD; não alternação periódica do oxímetro de pulso entre os dedos e do manguito de PA entre os membros.

Outro ponto relevante a ser destacado é a alta incidência de desenvolvimento de lesões por dispositivos respiratórios (53\%), sendo: TOT com lesões em boca, Fixação do TOT e Cateter Nasal tipo óculos com lesões em região de face, orelha e nariz. Fator que também contribuiu para elevada taxa de ocorrência de lesões associadas a dispositivos respiratórios foi o alto índice de Intubação Orotraqueal (IOT), sendo que dos 33 (100\%) pacientes analisados, $30(90,9 \%)$ foram submetidos à IOT em consequência ao acometimento pulmonar resultante da contaminação pelo SARS-CoV-2 ampliando os fatores de risco para o desenvolvimento dessas lesões devido sedação, imobilidade, utilização de drogas vasoativas e uso de diversos dispositivos médicos.

Em um estudo realizado na Turquia avaliando ocorrências de LPRDM envolvendo 175 pacientes internados em UTIs identificou-se $45 \%$ das lesões causadas pelo TOT, $10,4 \%$ por máscara de VNI, 7,1\% por máscara de $\mathrm{O} 2$ e 6,6\% por cânulas nasais, evidenciando o alto índice relacionado a dispositivos respiratónios (HANONU S E KARADAG A, 2016).

Dentre as lesões associadas a dispositivos respiratórios, destaca-se a predominância de lesões relacionadas à fixação do TOT. Segundo Black JM e Kalowes P (2016) o elevado índice dessa modalidade de lesão é atribuído a preocupação de manter o dispositivo firme e vedado ao paciente sem risco de deslocamento ao movimento, gerando fixações apertadas e consequentes lesões em regiões de boca, orelhas, columela nasal, face dentre outros locais.

As LPRDM associadas à SVD também ocupam um ranking relevante nesta pesquisa já que representam numericamente $16 \%$ das lesões manifestadas. Durante a coleta de dados foi observada a ausência de fixação desses dispositivos gerando lesões em região de coxa 7,4\% e em meato uretral $9 \%$ sendo mais predominante em pacientes do sexo feminino em região de coxa e no sexo masculino lesões em meato uretral, indicando necessidade de implantação de avaliação diária desse dispositivo e fixação adequada, em região de coxa para mulheres e em abdome inferior para homens, reduzindo assim a força de tração do dispositivo (BLACK JM e KALOWES P, 2016; GALETTO SGS, et al 2019).

Lesões por SNE foram evidenciadas em $12 \%$ dos casos, apresentadas majoritariamente em região de asa nasal associadas à tração excessiva do dispositivo. Além de ser um agente externo, este dispositivo médico geralmente é mantido por longo período de permanência podendo gerar desconforto e lesões. Este tipo de lesão não representa uma complicação grave, porém é considerada um evento adverso evitável através de 
boas práticas aplicadas. Estudo observacional prospectivo mostrou a incidência de lesão por pressão relacionada à SNG/SNE de 25,2\% sendo o principal motivo, sua fixação (MOTTA APG, et al., 2021). Segundo Asti $E$ (2017) as lesões relacionadas a este dispositivo são raramente citadas repres entando uma incidência que varia de $4,8 \%$ até $8 \%$, apresentando-se geralmente em forma de isquemia tecidual relacionado a fixação inadequada.

As lesões por Oxímetro de pulso representam 9\% e por Manguito de Pressão Arterial 10\% evidenciados em sua predominância nos pacientes com maior tempo de internação e devido uso prolongado no mesmo membro. Lesões por oxímetro de pulso também estão associadas em casos de pacientes em uso de drogas vasoativas com perfusão periférica prejudicada, sendo considerado fator de risco para desenvolvimento dessas lesões, neste caso indica-se o reposicionamento periódico destes dispositivos. Estudo realizado em UTIs na Turquia evidenciou um índice de $8 \%$ de LPRDM poroxímetro de pulso. Quanto a frequência de lesões por Manguito de Pressão Arterial, ainda é um tema limitado na literatura (HANONU S e KARADAG A, 2016; GALETTO SGS, et al., 2021).

O alto índice de LPRDM desenvolvidas em UTI podem estar diretamente associadas ao fato da equipe de enfermagem e os demais profissionais em saúde enfatizarem as patologias e demais órgãos e esqueceremse da pele. Diante disso, os pacientes se recuperam de sua patologia de base, porém levam consigo lesões decorrentes do período de internação por meses ou até anos. Em estudo realizado em uma UTI, foram identificadas 179 lesões por pressão adquiridas no hospital, dentre elas, 50 dessas lesões estavam relacionadas a dispositivos médicos. A prevalência e incidência de LPRDM em adultos internados em serviços de unidade de terapia intensiva são de $86 \%$ sendo as localizações mais encontradas a região da cabeça, região auricular, boca e em grande parte das mucosas (BARAKAT-JOHNSON M, et al., 2017).

Estudo realizado nos Estados Unidos sobre da prevalência de lesões por pressão com 104.266 pacientes, evidenciou uma taxa de $19,9 \%$ de LPRDM, enquanto $14,3 \%$ eram de LP em região sacral, $10,2 \%$ no calcâneo e 8,8\% nas nádegas. Estima-se que mais de um terço das lesões por pressão são adquiridas em hospitais e ainda relacionadas a dispositivos médicos (ASTI E, et al., 2017; CAVALCANTI EO e KAMADA I, 2020).

As LPPs representam um tema relevante no mundo inteiro e está ligado diretamente à qualidade da assistência prestada e a segurança do paciente através da RDC no 36 que "Institui ações para a segurança do paciente em serviços de saúde e dá outras providências" determinando aos serviços de saúde a criação do Núcleo de Segurança do Paciente (NSP) responsável pela elaboração do Plano de Segurança do Paciente em Serviços de Saúde (PSP) a fim de estabelecer diversas estratégias e ações de gestão de risco, como por exemplo a prevenção de úlceras por pressão (MINISTÉRIO DA SAÚDE, 2013a).

Diante das principais estratégias a serem implantadas na prevenção de lesões visando a segurança do paciente, está a Portaria o 2.095 de 24 de Setembro de 2013 que aprovou os protocolos básicos de segurança do paciente, dentre eles o "Protocolo para Prevenção de Úlceras por Pressão" com o objetivo de promover a prevenção da ocorrência de Úlceras Por Pressão (UPP) e outras lesões na pele distribuídos em 6 etapas: Avaliação de úlcera por pressão na admissão de todos os pacientes; Reavaliação diária de risco de desenvolvimento de UPP de todos os pacientes internados; Inspeção diária da pele; Manejo da Umidade: manutenção do paciente seco e com a pele hidratada; Otimização da nutrição e da hidratação e Minimizar a pressão (MINISTÉRIO DA SAÚDE, 2013b).

No que se refere aos cuidados na prevenção das LPRDM assemelham -se aos cuidados na prevenção de lesões citadas acima de modo geral, apresentando apenas algumas particularidades de cada dispositivo como a fixação adequada, escolha de tamanho e posicionamento do mesmo. Diante das evidências apresentadas é importante pontuar a necessidade da avaliação diária da equipe de enfermagem ao paciente crítico no processo de prevenção dessas lesões observando possíveis falhas no cuidado visando a segurança do paciente e qualidade na assistência prestada. As evidências indicam que as LPPs podem ser reduzidas a partir da execução de diretrizes e pacotes de cuidados, portanto, ter ciência da frequência dessas lesões na UTI e principais fatores de risco em potencial é o ponto chave para a aplicação de estratégias de prevenção (COYER F e TAYYIB N, 2017; CAVALCANTI EO e KAMADA I, 2020). 
As LPRDM estão associadas a uma gama de dispositivos utilizados em UTI e essas lesões são desafiadoras para as equipes de enfermagem, já que, apesar dos riscos causados pelo uso contínuo dos dispositivos médicos, os mesmos são componentes diagnósticos e terapêuticos essenciais na assistência ao paciente crítico. A enfermagem tem participação fundamental no desenvolvimento e aplicação de processos que facilitem e garantam uma assistência de qualidade atendendo aos protocolos de segurança do paciente (BLACK JM e KALOWES P, 2016; SILVA AT, et al., 2016).

A literatura aponta a escala de Braden como aliada na prevenção de LPRDM e apesar de não ser uma escala exclusiva para prevenção dessas lesões, se mostrou como um marcador sensível concluindo que os pacientes com escores altos desenvolveram menor índice de lesões, enquanto pacientes com menores escores desenvolveram maior prevalência de LPRDM (HANONU S e KARADAG A, 2016).

Diante disso, destaca-se a necessidade de ampliar o olhar ao paciente crítico trazendo em mente não apenas os riscos quanto às LPPs tradicionais por decúbito, mas também para o risco de desenvolvimento de LPRDM através da implantação e execução de medidas preventivas, tratamento adequado e avaliação diária de risco (GALETTO SGS, 2018).

Diante das recomendações gerais para a prevenção de LPRDM estão: Identificar dispositivos de risco para as LPRDM, escolher tamanho correto do dispositivo médico de acordo com as características do paciente; encaixar e fixar dispositivo adequadamente; avaliar diariamente a fixação do dispositivo e/ou método de fixação assim como verificar a tensão; avaliar pele sob o dispositivo médico diariamente; realizar limpeza e hidratação da pele íntegra; estar ciente quanto ao aspecto da pele e do risco potencial de ruptura da pele (ex: edema, pele úmida e fragilizada); atentar para que os dispositivos rígidos não sejam colocados diretamente sob o paciente acamado ou imóvel; aplicação de placas de hidrocolóide ou uso de gaze para alívio em pontos de pressão para prevenção; avaliar diariamente e questionar a necessidade de manter o uso do dispositivo ou se o mesmo não necessita ser substituído (EDSBERG LE, et al., 2016; ARNOLD-LONG M, et al., 2017; BARAKAT-JOHNSON M, et al., 2017; GALETTO SGS, et al., 2019; CAVALCANTI EO e KAMADA I, 2020).

Também representam ações para prevenção: mover ou reposicionar periodicamente dispositivo para avaliação da pele, bem como realizar alternação de dispositivos entre os membros a cada 2 horas para redistribuição da pressão; verificação cutânea a cada 3 ou 4 horas; promoção de educação continuada sobre utilização correta de dispositivos médicos e prevenção de lesões cutâneas (MINISTÉRIO DA SAÚDE, 2013b; ARNOLD-LONG M, et al., 2017; BARAKAT-JOHNSON M, et al., 2017; GALETTO SGS, et al., 2019; CAVALCANTI EO e KAMADA I, 2020).

Os cuidados de enfermagem no tratamento dessas lesões são: Identificar e especificar as LPRDM nos registros de enfermagem a fim de permitir a análise e solução da causa raiz do problema; evitar o posicionamento de dispositivo médico sobre locais com lesões; ajustar dispositivo em locais de pressão com ulceração pré-existente (ex: hiperemia e edema); realizar a limpeza da lesão com solução salina e realizar aplicação de cobertura indicada para evolução da mesma (EDSBERG LE, et al., 2016; ARNOLD-LONG M, et al., 2017; GALETTO SGS, et al., 2019).

Dentre as limitações deste estudo aponta-se o período da coleta de dados que coincidiu com a pandemia pelo SARS-CoV-2 e o alto risco de exposição. Ressalta-se também como limitação o recorte temporal de 5 anos para o referencial teórico, que excluiu assim, pesquisas sobre a temática publicadas antes deste período.

\section{CONCLUSÃO}

Conclui-se por meio dessa pesquisa o alto índice de ocorrência de LPRDM que indica a necessidade de intervenções de equipe de enfermagem para a redução das mesmas e melhora na qualidade da assistência, visto que, a equipe de enfermagem possui um papel imprescindível no processo do cuidar. Apesar deste tema ser pouco destacado na literatura quando comparado às lesões por decúbito, observam-se estratégias já publicadas com foco na redução das LPRDM. Diante disso, salienta-se a necessidade da aplicação das ferramentas e estratégias já existentes para avaliação dos fatores de risco e prevenção de LPRDM dentro das UTIs a fim de propiciar a redução destes eventos e melhora na qualidade de assistência de enfermagem. 


\section{REFERÊNCIAS}

1. ALI YCMM, et al. Incidence of pressure injury and nursing care time in intensive care. ESTIMA, Braz. J. Enterostomal Ther, 2020; 18: e1120.

2. ARNOLD-LONG M, et al. Medical device-related pressure injuries in long-term acute care hospital setting. Journal of Wound, Ostomy and Continence Nursing, 2017;44(4):325-330.

3. ASTI E, et al. Prevalence and risk factors of nasal pressure ulcers related to nasogastric intubation: an observational study. European Surgery - Acta Chirurgica Austriaca, 2017;49(4): 171-174.

4. BARAKAT-JOHNSON M, et al. Medical device-related pressure injuries: An exploratory descriptive study in an acute tertiary hospital in Australia. Journal of Tissue Viability, 2017;26(4): 246-253.

5. BEZERRA SMG, et al. Nursing strategies for pressure injury prevention in surgical patients. ESTIMA, Braz. J. Enterostomal Ther. 2020; 18: e1020.

6. BLACK JM, KALOWES P. Medical device-related pressure ulcers. Chronic Wound Care Management and Research, 2016;3: 91-99.

7. BORGHARDT AT, et al. Pressure ulcers in critically ill patients: incidence and associated factors. Rev. Bras. Enferm, $2016 ; 69(3): 460-467$.

8. CAVALCANTI EO, KAMADA I. Medical-device-related pressure injury on adults: An integrative review. Texto e Contexto Enfermagem, 2020;29:1-14.

9. COYER F, TAYYIB N. Risk factors for pressure injury development in critically ill patients in the intensive care unit: a systematic review protocol. Systematic Reviews, 2017, 6: 58.

10. DELMORE BA, AYELLO EA. Pressure Injuries Caused by Medical Devices and Other Objects: A Clinical Update. AJN -American Journal of Nursing, 2017;117(12):36-45.

11. DONOSO MTV, et al. Cost Analysis Of The Treatment Of Pressure Injury In Hospitalized Patients. Revista de Enfermagem do Centro-Oeste Mineiro, 2019;9 (e3446):12.

12. EDSBERG LE, et al. Revised National Pressure Ulcer Advisory Panel Pressure Injury Staging System. Journal of Wound, Ostomy and Continence Nursing, 2016;43(6):585-597.

13. GALETTO SGS. Lesões por pressão relacionadas a dispositivos médicos em pacientes críticos: características clínicas e o olhar da enfermagem. Tese (doutorado) - Universidade Federal de Santa Catarina, Centro de Ciências da Saúde, Programa de Pós-Graduação em Enfermagem, Florianópolis, 2018;221p.

14. GALETTO SGS, et al. Medical Device-Related Pressure Injuries: an integrative literature review. Rev Bras Enferm, 2019;72(2): 528-36.

15. GALETTO SGS, et al. Medical device-related pressure injury prevention in critically ill patients: nursing care. Rev Bras Enferm. 2021;74(2): e20200062.

16. HANONU S, KARADAG A. A Prospective, Descriptive Study to Determine the Rate and Characteristics of and Risk Factors for the Development of Medical Device-related Pressure Ulcers in Intensive Care Units. Ostomy Wound Manage, 2016;62(2): 12-22.

17. KAYSER SA, et al. Prevalence and Analysis of Medical Device-Related Pressure Injuries: Results from the International Pressure Ulcer Prevalence Survey. Adv Skin Wound Care, 2018; 31:276Y85.

18. MOTTA APG, et al. Nasogastric/nasoenteric tube-related adverse events: an integrative review. Rev. Latino-Am. Enfermagem 2021;29:e3400.

19. MINISTÉRIO DA SAÚDE. Rdc n 36 de 25 de julho de 2013: Institui ações para a segurança do paciente em serviços de saúde e dá outras providências. Brasil, 2013a. Disponível em: https://bvsms.saude.gov.br/bvs/saudelegis/anvisa/2013/rdc0036_25_07_2013.html. Acesso em: 10 de junho de 2021.

20. MINISTÉRIO DA SAÚDE. Protocolo para prevenção de úlcera por pressão. 2013b. Acesso 10 jun 2021. Disponível: https://www.hospitalsantalucinda.com.br/downloads/prot_prevencao_ulcera_por_pressao.pdf. Acesso em: 10 de junho de 2021.

21. SILVA AT, et al. Nursing care and the focus on patient safety in the Brazilian scenario. Saúde Debate |Rio de Janei ro, 2016;40(111):292-301. 